\title{
A synthetic curcuminoid derivative inhibits nitric oxide and proinflammatory cytokine synthesis
}

\begin{abstract}
Curcumin is a highly pleiotropic molecule with significant regulatory effects upon inflammation and inflammatory related diseases. However curcumin has one major important limitation in which it has poor bioavailability. Design of synthetic structural derivatives of curcumin is but one approach that has been used to overcome its poor bioavailability while retaining, or further enhancing, its drug-like effects. We have synthesized a series of curcumin analogues and describe the effects of 2,6-bis-4-(hydroxyl-3-methoxy-benzylidine)cyclohexanone or BHMC upon nitric oxide and cytokine synthesis in cellular models of inflammation. BHMC showed a significant dose-response inhibitory action upon the synthesis of NO and we have shown that this effect was due to suppression of both iNOS gene and enzyme expression without any effects upon scavenging of nitrite. We also demonstrated that BHMC has a very minimal effect upon iNOS activity with no effect at all upon the secretion of PGE(2) but has a strong inhibitory effect upon MCP-1 and IL-10 secretion and gene expression. Secretion and gene expression of TNF-alpha and IL-6 were moderately inhibited whereas IL-8 and IL-1beta were not altered. We conclude that BHMC selectively inhibits the synthesis of several inflammatory mediators. BHMC should be considered a promising drug lead for preclinical and further pharmacological studies.
\end{abstract}

Keyword: Cytokine; Chemokine; INOS; Curcuminoid; Macrophage 